\title{
Synchronizing actions with events: The role of sensory information
}

\author{
GISA ASCHERSLEBEN \\ Max-Planck-Institut, Munich, Germany \\ and \\ WOLFGANG PRINZ \\ Max-Planck-Institut, Munich, Germany \\ and Ludwig-Maximilians-Universität, Munich, Germany
}

\begin{abstract}
Tasks requiring the subject to tap in synchrony to a regular sequence of stimulus events (e.g., clicks) usually elicit a response pattern in which the tap precedes the click by about $30-50$ msec. This "negative asynchrony" was examined, first, by instructing subjects to use different effectors for tapping (hand vs. foot; Experiments 1 and 2), and second, by administering extrinsic auditory feedback in addition to the intrinsic tactile/kinesthetic feedback (Experiment 2). Experiment 3 controlled whether the results observed in Experiment 2 were due to purely sensory factors within the auditory modality. Results suggest that taps are synchronized with clicks at the central level by superimposing two sensory codes in time: the tactile/kinesthetic code that represents the tap (the afferent movement code) and the auditory code that represents the click (the afferent code that results from the guiding signal). Because the processing times involved in code generation are different for these two central codes, the tap has to lead over the click.
\end{abstract}

Sensorimotor synchronization tasks require the subject to perform certain movements in synchrony with certain events. Such tasks are fairly easy, provided that the timing of the pertinent events is perfectly predictable. A simple means of ascertaining this is to present subjects with a sequence of periodically repeated, equidistant stimuli and to require them to perform some movement in synchrony. For instance, in Stevens's classical study, subjects listened to a sequence of metronome beats and had to accompany the sequence of beats with a corresponding sequence of synchronous keypressings (Stevens, 1886).

In spite of their seeming triviality, periodic synchronization tasks have attracted the attention of researchers in action control for a long time. In particular, two aspects of task performance have been studied in detail: The first concerns the control of the timing of a periodic sequence of motor acts. In this line of research, the central issue is to model the mechanisms that generate and maintain a periodic sequence of motor acts. Typically, the analysis of this aspect of performance relies on data that reflect properties of the time series of the motor acts

\footnotetext{
We wish to thank Frank Miedreich for programming; Esther Löb for collecting the data; Jonathan Harrow and Heidi John for correcting our English; and Arthur Kramer, Jiři Mates, and an anonymous reviewer for their helpful criticism, suggestions, and comments on an earlier draft. Inquiries or requests for reprints should be sent to G. Aschersleben, Max-Planck-Institut für psychologische Forschung, Postfach 4401 09, 80750 München, Germany (e-mail: aschersl@mpipf-muenchen. mpg.de).
}

(intertap intervals and their autocorrelation pattern), and there iz less interest in the phase relationships between the stimulus sequence and the motor sequence. In many studies, the stimulus sequence is even used as an experimental tool for parameterizing the sequence of motor acts in a desired way. Once these parameters have been set, the timing of the motor acts can be studied after the pacing stimulus is switched off-that is, in the continuation phase of the synchronization task (see, e.g., Collyer, Broadbent, \& Church, 1992, 1994; Ivry, Keele, \& Diener, 1988; Kolers \& Brewster, 1985; Mates, 1991; Povel, 1981; Stevens, 1886; Summers, Bell, \& Burns, 1989; Vorberg \& Hambuch, 1978, 1984; Vos \& Ellermann, 1989; Wing, 1977, 1982; Wing \& Kristofferson, 1973a, 1973b; Yamanishi, Kawato, \& Suzuki, 1979, 1980).

The second aspect focuses on that which is disregarded by the first: the coupling between (perceived and/or anticipated) stimulus events and (performed) motor acts. In this line of research, the central issue is to model the mechanism that induces a periodic pattern of motor acts in synchrony with a corresponding pattern of stimuli. How precisely can synchrony between stimulus and motor events (say, metronome beats and keypresses, or clicks and taps) be achieved, and which factors determine this precision? Are there any systematic deviations from synchrony, and how can they be explained? As one might expect, the analysis of these aspects of performance relies on data that reflect the temporal relationship between stimulus events and motor acts (e.g., the asynchrony between the onsets of the clicks and the onsets of the taps). 
This study addresses the issue of coupling in sensorimotor synchronization. In the experiments reported below, we began with a well-established experimental effect and sought to understand the mechanisms underlying it. The effect is the "negative synchronization error," or "negative asynchrony," which is usually observed in periodic synchronization tasks: The tap leads over the click by a certain amount of time, ranging somewhere between 20 and $50 \mathrm{msec}$, depending on task conditions and subjects. Negative asynchrony was first observed by Dunlap (1910) and was replicated in several later studies (see, e.g., Fraisse, 1966, 1980; Fraisse, Oléron, \& Paillard, 1958; Franek, Radil, Indra, \& Lánsky, 1987; Hary \& Moore, 1985; Kolers \& Brewster, 1985; Mates, Radil, \& Pöppel, 1992; Peters, 1989; Truman \& Hammond, 1990; Vos \& Helsper, 1992; Woodrow, 1932; but see also Schulze, 1992, and Najenson, Ron, \& Behroozi, 1989, for two studies in which the effect was not observed; for a review see Aschersleben, 1994).

An interesting hypothesis to account for the effect was proposed during the 1980 s. It was first published by Fraisse (1980), who credited it to Paillard, so that it may aptly be termed the Paillard-Fraisse hypothesis (see also Paillard, 1949). Some years later, a similar explanation of synchronization errors was also offered by Hary and Moore (1985), and recently, it was picked up by Mates (1994a, 1994b), who integrated the idea in a more general model on synchronization. According to the Paillard-Fraisse hypothesis, the brain synchronizes taps with clicks superimposing two sensory codes in time: the auditory code representing the click, and the tactile/kinesthetic code representing the tap. However, if the tap code is to coincide with the click code (in the brain), then the tap and the click themselves cannot coincide (in the world). This is because the processing times involved in code generation are different for these two central codes. For example, for bare anatomical reasons, it takes more time for sensory information to travel from the fingertip to the brain than from the ear to the brain (see Figure 1).

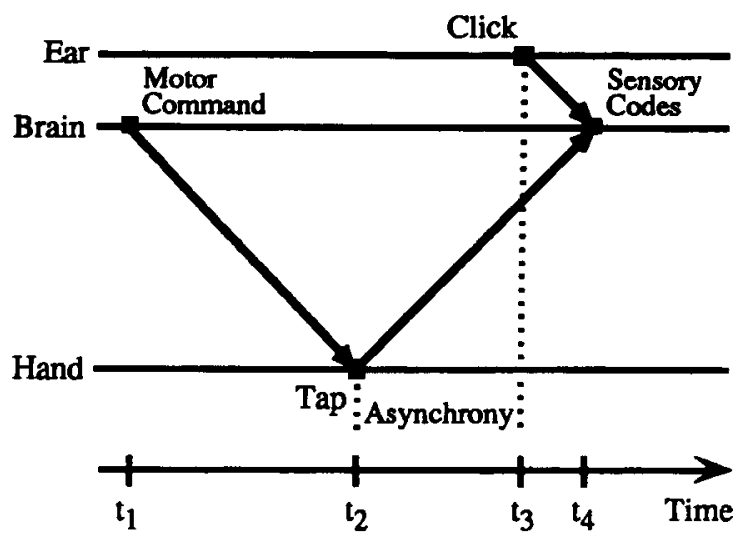

Figure 1. Illustration of the Paillard-Fraisse hypothesis. The distances between the horizontal lines indicate the different functional distances between the ear and the brain and between the hand and the brain (modified after Prinz, 1992).
Hence, in order to achieve temporal coincidence of the two codes (in the brain), the tap has to lead over the click (in the world) by an amount of time that corresponds to the difference between the two code generation times. A system like this, which synchronizes world events by means of synchronizing their brain codes, cannot avoid synchronization errors by principle. As long as it keeps to the principle of coincidence of internal codes, it has no way of achieving veridical coincidence of external events (even if it had precise knowledge of the code generation times involved).

This account has two notable theoretical implications: First, it entails the notion of an isomorphic relationship between the perception of temporal order and the temporal order of the brain codes that perception is based on. If one believes that perceived coincidence of clicks and taps is grounded on the physical coincidence of their respective brain codes, one is basically claiming that the temporal order of mental events is isomorphic with the temporal order of the underlying brain events. Note that this view relies on the "Cartesian Theater" model of the mind that has recently been attacked by Dennett and Kinsbourne (1992). Second, it stresses the role of sensory, as opposed to motor, codes in assuming that it is the sensory code of the action (tap) that must be made to coincide with the sensory code of the external event (click). According to this view, synchrony is achieved and maintained at the level of perceived events, that is, by synchronizing the perceived tap with the perceived click (and it is implied that there is no problem involved in timing the motor commands in such a way that coincidence of the two sensory codes is achieved).

Regarding the latter aspect, the Paillard-Fraisse hypothesis shares a basic theoretical assumption with the framework provided by the common coding approach to perception and action (Prinz, 1990, 1992; for related views see, e.g., Allport, MacKay, Prinz, \& Scheerer, 1987; Greenwald, 1970; Meltzoff, Kuhl, \& Moore, 1991). This approach posits a common representational domain for the representational structures involved in perception and in action control. In particular, it holds that the codes that underlie the perception of events can also be functional in the control of actions (implying that actions are planned and controlled in terms of their perceivable effects). The Paillard-Fraisse hypothesis entails a specific application of this general logic to the time domain, in that it assumes that the perception of clicks and the generation of taps rely on a shared time axis and that taps are generated in such a way that perceived synchrony is achieved.

Some further accounts of synchronization errors have occasionally been discussed in the literature. Three major hypotheses should be mentioned: the $P$-center hypothesis, the evaluation hypothesis, and the undershooting hypothesis. According to the P-center hypothesis, the functional onset of the perceived event may be different from its nominal onset, depending on various factors such as the duration of the event, the abruptness of its onset, and so forth (Marcus, 1976; Morton, Marcus, \& Frankish, 
1976; Schütte, 1978a, 1978b; Terhardt \& Schütte, 1976). If this is the case, it can be reasonably argued that clicks and taps differ with respect to these factors. Clicks are quasi-punctiform events with abrupt onsets and offsets, so that there cannot be much difference between functional and nominal onsets. This is different with taps, which are extended in time, and their onsets tend to be less sharp and less clearly defined. As a consequence, their functional onsets could lag behind their nominal onsets, and if synchrony is achieved by superimposing the functional onsets of the two events, a negative asynchrony should arise (Auxiette, 1992; Gérard \& Auxiette, 1992). One possible prediction from this hypothesis is that the size of the asynchrony should be positively correlated with tap duration: The longer the tap, the larger the displacement of the functional onset relative to the nominal onset should be, this giving rise to the asynchrony.

The two remaining hypotheses locate the effect in different parts of the timekeeping mechanism believed to underlie periodic synchronization. The evaluation hypothesis holds that negative asynchronies arise in the error correction component of the timekeeper as a result of an asymmetric evaluation of positive and negative errors. This asymmetric evaluation follows the principle that being early is better than being late, so that positive errors (being late) are more strongly corrected than negative ones (being early). This possibility has incidentally been mentioned in the literature, by, for example, Vos and Helsper (1992) and Vos, Helsper, and van Krysbergen (1992). As Koch (1992) has recently shown, the size of the asynchrony that results under a given asymmetric evaluation function must depend on the variance of the mechanism controlling the generation of taps. The larger this variance is, the more will the mean of the tap distribution be antedated relative to the pacing stimulus.

Finally, the undershooting hypothesis locates the effect in the timekeeping mechanism itself-more precisely, in the operations by which the parameters are fixed. It is based on the observation that the variance of biological timekeepers tends to be positively correlated with the intervals to be controlled. Therefore, in any given task, these timekeepers can reduce their variance by systematically undershooting their temporal goals. As has recently been argued by Vorberg and Wing (1993), a mechanism like this could also be responsible for the occurrence of negative synchronization errors in periodic synchronization.

The experiments reported below were planned to test the validity of the Paillard-Fraisse hypothesis. Accordingly, they are based on the logic of this hypothesis so that clear-cut predictions can only be derived from this account. Yet, as far as it is available, we will also report the pertinent evidence for the competing accounts and come back to their evaluation in the General Discussion.

Up till now, the empirical basis for the Paillard-Fraisse account is rather weak. Though Fraisse (1980) has reported a few preliminary experiments that lend some support to it, his results are not very conclusive, because of insufficient detail in reporting the experiments and results (cf. the appendix to Fraisse, 1980). Some more re- cent evidence comes from Pöppel, Müller, and Mates (1990), Mates et al. (1992), and Vos and Helsper (1992). Their experiments refer to the account as an explanation for the negative asynchrony, but without putting it to critical tests. The logic underlying the Paillard-Fraisse hypothesis has also been taken up recently in two studies by Bard et al. (1992) and Bard, Paillard, Teasdale, Fleury, and Lajoie (1991), to account for the phase relationships between two effectors involved in synchronous movements.

The present study was designed to collect further evidence pertinent to this account and to test and elaborate it in a more systematic way. Though they differ in the factors manipulated, the following experiments share a simple methodological principle. As can be derived from Figure 1, one of the obvious implications of the PaillardFraisse hypothesis is that the size of the (negative) asynchrony depends on the time elapsing between the onset of the motor act in the world (at $t_{2}$ ) and the onset of the central code representing that act in the brain (at $\left.t_{4}\right)$. The longer this interval, the longer the motor act (tap) must lead over the stimulus event (click) in order to achieve coincidence of the two brain codes.

We manipulated this interval in different ways: First, following Fraisse (1580), we instructed subjects to use different effectors for tapping, entailing different times for the generation of central codes on the basis of sensory stimulation (Experiments 1 and 2). Second, we administered extrinsic auditory feedback in addition to the intrinsic tactile/kinesthetic feedback arising from the taps. This was done in an attempt to shift the onset of tap-generated central codes to an earlier point in time (Experiment 2). In Experiment 3, which was designed as a control experiment, we tested whether the results observed in Experiment 2 might be due to purely sensory factors within the auditory modality.

\section{EXPERIMENT 1}

Fraisse (1980) studied synchronization under conditions in which a movement had to be performed either with the hand or with the foot. He observed a negative asynchrony between stimulus onset and response onset when the tap was produced by the hand and an even larger asynchrony when the tap was produced by the foot. More specifically, there were two conditions: (1) synchronization of either hand or foot tapping with an auditory pacing signal, and (2) synchronization of hand and foot tapping with an auditory pacing signal. In the two conditions, it was found that the tap of the hand led over the signal by about $50 \mathrm{msec}$. When the two effectors had to perform the movement simultaneously, the foot led over the hand by another $20 \mathrm{msec}$. When each effector was involved individually (Condition 1) the difference in asynchrony between hand and foot tapping was about $11 \mathrm{msec}$. Fraisse interpreted these results as a support for his view that simultaneity of the response and the stimulus is controlled at a central level. This would explain why the reaction at the peripheral level is per- 
formed earlier when the distance between the effector and the cortex is larger.

Paillard (1949; for a replication, see Bard et al., 1992) also studied tapping tasks with "voluntary movements"that is, subjects had to move two effectors (right or left hand, right or left foot) simultaneously under conditions in which they self-paced their movement onsets. In conditions with synchronized tapping with one hand and one foot, he observed a lead of the foot movement over the hand movement. In symmetric conditions, in which both hands or both feet tap simultaneously, subjects were able to synchronize their tap onsets almost exactly.

Our main goal in Experiment 1 was to replicate Fraisse's results under more controlled conditions. A second goal was to find out whether the asynchrony is affected by the body side of the limbs involved. Accordingly, three factors were varied: the effector that performs the movement (hand vs. foot), the body side (right vs. left), and the effector coupling (single vs. coupled). In the coupled conditions, all three possible combinations of pairs of limbs were studied: horizontal (simultaneous tapping by the two hands or the two feet), vertical (right hand and right foot or left hand and left foot), and diagonal (right hand and left foot or left hand and right foot). If the Paillard-Fraisse hypothesis holds, negative asynchronies should be observed under all conditions. Furthermore, since it takes more time to generate a sensory code from a foot tap in comparison with a hand tap, asynchronies should be larger for foot movements than for hand movements throughout. There is no particular prediction concerning body side and effector coupling. Basically, it was expected that these factors would not affect the size of the asynchrony.

\section{Method}

Subjects. Originally, the sample consisted of 14 subjects who reported that they were right-handed. Two subjects had to be excluded from the final analysis because they did not conform to the preset criteria for acceptable performance (see below). The remaining 12 subjects ( 7 females and 5 males, mean age 25 years) reported no sensory defects and were naive with regard to the experiment's purpose. To control the influence of handedness, we kept this factor constant by using only right-handed subjects.

Apparatus and Stimuli. The subject was seated at a table in a sound-absorbing room. He/she was asked to tap with the index finger or the big toe on a silent electrical contact switch. A wooden box covered the response apparatus, and the responding effector eliminating visual feedback. The auditory signal $(400 \mathrm{~Hz}, 82 \mathrm{~dB}[\mathrm{~A}])$ was presented binaurally through headphones (AKG K240, 600 $\Omega$ ). The visual warning signal was presented by a red/green LED. To cover external sounds, white noise (53 dB[A]) was used under all conditions. The auditory stimuli were produced by a personal computer (Hewlett-Packard Vectra QS/20) via a D/A converter and an amplifier (Sony TA-F170). The computer controlled the experimental procedure and registered the onset and the duration of keypresses (with a resolution of $1 \mathrm{msec}$ ).

Procedure. There were two experimental sessions. In the first session, the coupled conditions were administered. Subjects were asked to tap with two effectors simultaneously in the three subconditions horizontal, vertical, and diagonal. Two tasks in each subcondition differed in the pair of effectors involved.
To avoid carryover effects across conditions, the second session was run 6 weeks later. In this session, subjects performed the four tasks contained in the single condition, in which only one of the four effectors performed the movement. As a control, the two tasks from the horizontal subcondition were also repeated in the second session.

Each session consisted of seven blocks. In the first block, subjects ran six practice trials to get used to the apparatus, each task being presented once. In the following six blocks, the experimental data were collected. For the six tasks in each session, six balanced orders of tasks were used, corresponding to a Latin square. Subjects were assigned to one of these orders at random and for both sessions separately. Each session was performed in the same order of tasks by 2 subjects respectively. Within each task, four practice trials were presented followed by eight test trials. Each trial contained the following sequence of events: First, a green light signaled that the trial could be started. After pressing a key, the subject was continuously exposed to white noise, and the sequence of the pacing signals was started (26 signals of $10 \mathrm{msec}$ each with an SOA [stimulus onset asynchrony] of $800 \mathrm{msec}$ ). The subject's task was to start tapping within the first three signals and then to tap along with the signal with the index finger and/or the big toe as precisely as possible. The instruction stressed onset synchronization; that is, subjects were required to synchronize the touch of the response key (either by one or by two effectors simultaneously) with the appearance of the click.

Data analysis. Data analysis started with the seventh signal in each trial. The first taps were not included, because a minimum of 3 to 5 signals was required to pick up the beat. Hence, the means, standard deviations, and key-touching times reported below always refer to the taps matching the remaining 20 signals in each trial. The means and standard deviations of asynchronies between tap onsets and click onsets and means of key-touching times were computed per trial. Negative values indicate that taps come first. Trials were eliminated when they contained less than 10 productions or when the standard deviation exceeded a criterion of $100 \mathrm{msec}$. A subject was dropped from the analysis when more than $25 \%$ of his/her trials had to be rejected according to these criteria.

Mean asynchronies per trial were entered into two repeated measurement analyses of variance (ANOVAs). The first $2 \times 2 \times 2 \times 8$ ANOVA examined four within-subject factors: the effector performing the movement (hand vs. foot), body side (right vs. left), task complexity (single effector vs. coupled effectors), and repetition within each condition ( 8 trials). Note that this analysis disregards the three different subconditions in the coupled condition, which were entered into the second ANOVA. This $2 \times 2 \times 3 \times 8$ ANOVA distinguished four within-subject factors within the coupled condition: the effector (hand vs. foot), body side (right vs. left), coupling (coupling condition, in which a given effector was used: horizontal vs. vertical vs. diagonal), and repetition within each condition ( 8 trials). Furthermore, pairwise comparisons were conducted with the Scheffé test. The level of significance was set at $p<.01$.

\section{Results}

First of all, $5 \%$ of the trials had to be rejected, because they failed to meet the criteria presented above. We then checked whether the absolute level of asynchrony was comparable across the two sessions. Since the $t$ test comparisons of the two identical subconditions (Horizontal 1 in the first session and Horizontal 2 in the second session) showed no significant effect at all $\left[t_{\text {hand }}(11)=\right.$ $\left.0.62, t_{\text {foot }}(11)=0.54\right]$, we concluded that the data from the two experimental sessions could be combined.

As expected, a negative asynchrony was observed throughout. The asynchrony was clearly negative when 
the hand performed the movement $\left(\bar{X}_{\text {hand }}=-50 \mathrm{msec}\right.$, $s=20 \mathrm{msec}$ ). In addition, the absolute value of the asynchrony was substantially larger in the foot condition $\left(\bar{X}_{\text {foot }}=-95 \mathrm{msec}, s=31 \mathrm{msec}\right.$ ) than in the hand condition. There was no effect of the effectors' body side (left vs. right) or of the number of limbs involved (single vs. coupled). Moreover, there was no difference in the size of the asynchrony between the three coupling conditions (see Figures 2 and 3 ). In both ANOVAs, only one source of variance was highly significant: the main effect of the factor effector [ANOVA $1, F(1,11)=111.13, p<$ .001 ; ANOVA $2, F(1,11)=68.90, p<.001] .{ }^{1}$ The Scheffé test revealed a critical difference of $14 \mathrm{msec}(p<.01)$.

The standard deviations of the asynchronies were larger for the foot than for the hand condition (foot, $39 \mathrm{msec}$; hand, $37 \mathrm{msec}$ ). Moreover, they were larger for left effectors than for right effectors (left, $39 \mathrm{msec}$; right, $37 \mathrm{msec}$ ), and they tended to decrease more in the coupled than in the single conditions (single, $40 \mathrm{msec}$; coupled, $36 \mathrm{msec}$ ). Within the coupled condition, the smallest standard deviation was observed in the diagonal subcondition (horizontal, $37 \mathrm{msec}$; vertical, $37 \mathrm{msec}$; diagonal, $34 \mathrm{msec}$ ).

Key-touching times were substantially larger in the foot than in the hand condition (foot, $215 \mathrm{msec}$; hand, $134 \mathrm{msec}$ ). Furthermore, they were longer in coupled than in single tasks (coupled, $189 \mathrm{msec}$; single, $160 \mathrm{msec}$ ), and left effectors showed longer keypresses than did right effectors (left, $182 \mathrm{msec}$; right, $166 \mathrm{msec}$ ). Concerning the three coupling subconditions, we observed an interaction with the factor effector: in the horizontal subcondition, the key touching time for the hand was smaller than in the other two subconditions (horizontal [hand], $130 \mathrm{msec}$; vertical [hand], $171 \mathrm{msec}$; diagonal [hand], $178 \mathrm{msec}$ ), while there was no such difference for the foot.

\section{Discussion}

The results of this first experiment can be viewed as a support for the Paillard-Fraisse hypothesis. This hypothesis predicts that the negative asynchrony will depend on the effector used but not on body side and coupling. That is exactly what we observed in this experiment. Like Fraisse (1980), we found an increase in the absolute amount of asynchrony when the foot performed the movement as opposed to the hand. However, in the present experiment, the magnitude of this effect ( $45 \mathrm{msec}$ ) was clearly larger than in Fraisse's (1980) study $(16 \mathrm{msec})$. The EEG literature reports a difference between hand and foot tapping of about $50 \mathrm{msec}$ (thus supporting our results). For example, Shibasaki, Barrett, Halliday, and Halliday (1981) measured evoked potentials when subjects tapped with the hand or the foot. The "post-motion frontal negativity," which can be interpreted as an indicator for kinesthetic feedback, showed a latency of about $50 \mathrm{msec}$ for the foot movement compared to the hand movement.

The same amount of negative asynchrony is observed when two corresponding effectors (both hands or both feet) tap simultaneously. Upon first view, the fact that body side has no effect on the asynchrony seems surprising, implying that the size of the asynchrony is not affected by hand dominance (all subjects were right-handed). This observation, however, is consistent with the literature: In studies of the effect of side on tapping, no effect on the asynchrony has been observed under comparable experimental conditions (Ilmberger, Müller, Pöppel, Mates, \& Radil, 1990; Truman \& Hammond, 1990).

Furthermore, no effect of the repetitions is observed; that is, after a warming-up phase, there is no change of asynchrony during the experiment. This also holds for the variation of asynchrony within each trial: the standard deviation per trial is constant from the beginning.

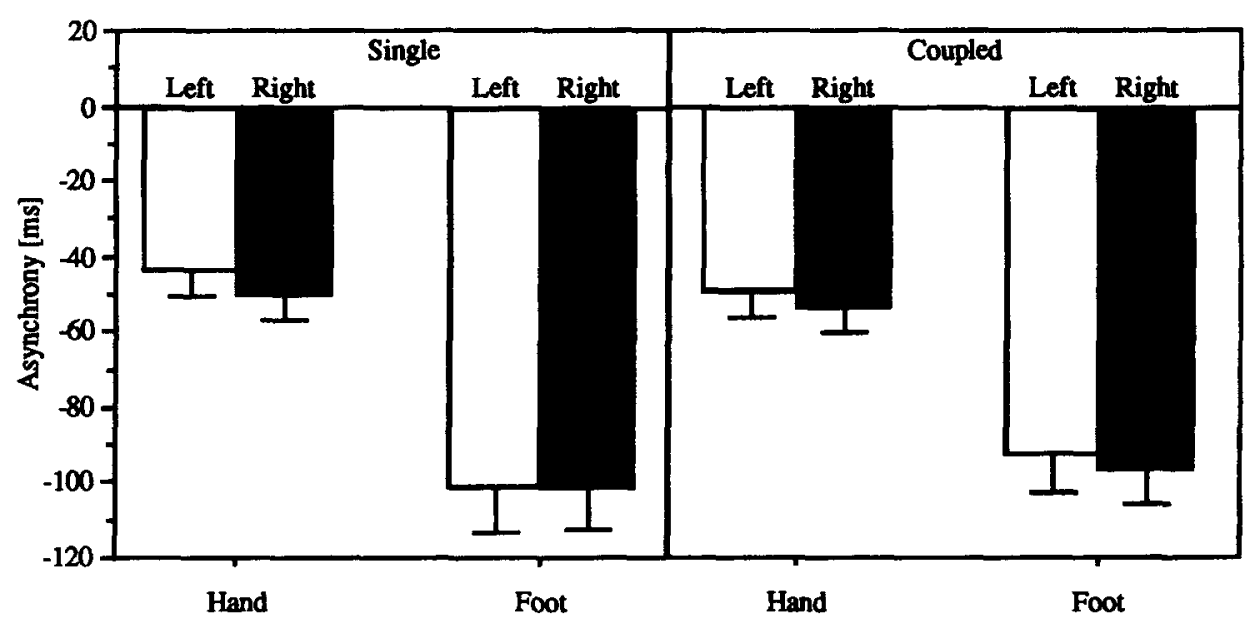

Figure 2. Mean asynchronies (and standard errors between subjects) under two effector conditions (hand vs. foot) and two body side conditions (right vs. left). The left graph shows the values for the single condition (one effector performs the movement); the right graph shows the values for the coupled condition (pairs of effectors perform the movement simultaneoushy). 


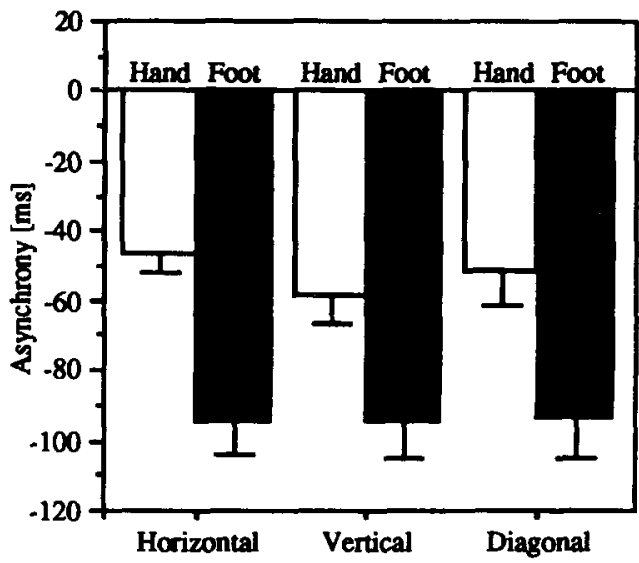

Figure 3. Mean asynchrony (and standard error between subjects) under two effector conditions (hand vs. foot) and three coupling conditions (horizontal vs. vertical vs. diagonal).

The other two dependent variables (standard deviations of asynchronies and key-touching times) will be considered in the General Discussion.

\section{EXPERIMENT 2}

In the first experiment, tactile/kinesthetic feedback was the sole source of information about the tap, since visual and auditory feedback was excluded. The second experiment was designed to study how asynchrony would be affected if auditory feedback was provided in addition to tactile/kinesthetic feedback. In this case, information about the tap is contained in two different sensory codes that will reach the brain at different points in time. There will be an early auditory code in addition to the late tactile/kinesthetic code.

Given the logic underlying the Paillard-Fraisse hypothesis, one out of three things might occur (see Figure 4): On the one hand, one might expect that the auditory feedback, which arrives first, takes the role of the central code that becomes superimposed on the central click code (at $t_{1}$ ), and that the tactile/kinesthetic feedback is completely irrelevant. In this case, the asynchrony should disappear completely, because the generation of the auditory feedback code requires exactly the same amount of time as the generation of the auditory click code. On the other hand, one could also expect the reverse: that only tactile/kinesthetic feedback can be functional in this way (at $t_{2}$ ), and that the auditory feedback is completely disregarded. In this case, the asynchrony should be unaffected by the additional auditory feedback signal. Finally, as a third option, one could also consider the possibility that the two tap-related codes enter into a joint-event code whose effective onset point lies somewhere between the onset of the early and the late feedback code; for example, it cuts the temporal distance between the two feedback codes in half (at $t_{3}$ ). In this case, one would expect that auditory feedback serves to reduce the size of the asynchrony but does not reduce it to zero.

A similar pattern could also emerge if different feedback signals were functional in different trials, so that in some trials the (early) auditory signal and in other trials the (late) tactile/kinesthetic feedback signal would take the role of the critical central code. If this is the case, a reduction of asynchrony would have to be expected, but this mixture of trials should, in addition, lead to an increase in variance relative to the control condition where no auditory feedback is available.

When auditory feedback is provided, a negative asynchrony is usually observed as well (Fraisse et al., 1958; Franek et al., 1987; Hary \& Moore, 1987; Mates et al., 1992; Oléron, 1961; Woodrow, 1932). In one of these studies, in which the two conditions with and without auditory feedback could be compared, a decrease of asynchrony was observed when additional auditory feedback was provided (Mates et al., 1992). This seems to support the third of the three solutions.

Two factors were manipulated in the present experiment: the feedback available to perform the task (tactile/kinesthetic [tk] vs. additional auditory feedback $[t k+a]$ ) and the effector (hand vs. foot). Concerning the influence of the effector under conditions with auditory feedback, again, one out of three things might occur (see Figure 4): If the tactile/kinesthetic feedback is completely irrelevant and the auditory feedback takes the role of the central code that becomes superimposed on the central click code, an asynchrony should be observed in neither the hand nor the foot condition. Therefore, the difference between hand and foot tapping should disappear in the auditory feedback condition. If, on the other hand, the auditory feedback is completely disregarded, the difference between hand and foot tapping should be the same under both feedback conditions. If the third solution is true-that is, if the two tap-related codes enter

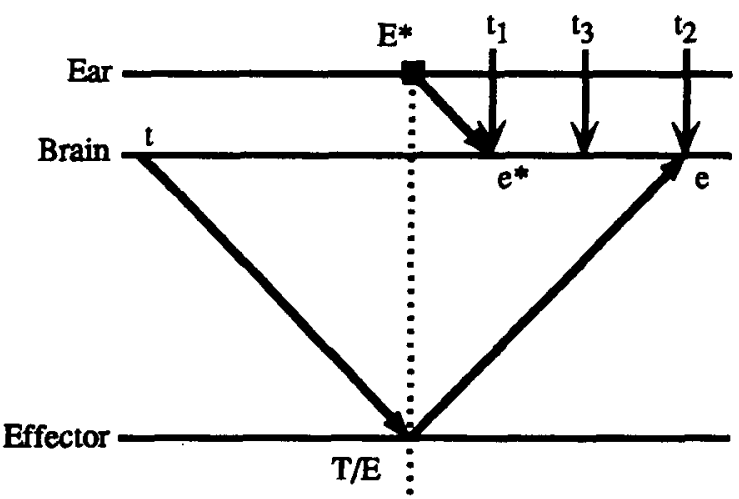

Figure 4. Temporal relations between the onset of the tap plus the auditory feedback and the corresponding central codes under conditions with additional auditory feedback. $T=$ tap; $E, E^{*}=$ effects resulting from the tap $\left(E\right.$, tactile/kinesthetic feedback; $E^{*}$, auditory feedback); $t, e, \mathrm{e}^{\star}=$ corresponding central codes; $t_{1}, t_{2}, t_{3}=$ three possible points in time when the feedback is functional for synchronization at the central level. 
into a joint-event code whose effective onset point lies between the onset of the early and the late feedback code-there should be an interaction between the feedback and the effector. The difference between the asynchronies observed under the two feedback conditions should be smaller for hand tapping than for foot tapping. This is because the amount of asynchrony reduction due to auditory feedback is, under this view, correlated positively with the absolute size of the asynchrony (and, hence, larger for foot than for hand tapping).

\section{Method}

Subjects. The original sample consisted of 16 new subjects. Two subjects had to be excluded from the final analysis, because they did not reach the preset criteria for acceptable performance (see Experiment 1). The remaining 14 subjects ( 7 females and 7 males, mean age 27.5 years) reported that they were right-handed and had normal auditory acuity; they were naive with regard to the experiment's purpose.

Apparatus and Stimuli. The apparatus and stimuli were the same as in Experiment 1. The additional auditory feedback $(2000 \mathrm{~Hz}$, $82 \mathrm{~dB}[\mathrm{~A}]$; duration, $10 \mathrm{msec}$ ) was presented binaurally through headphones.

Procedure. The subjects participated in 2 experimental sessions on 2 consecutive days. In one session, they tapped with the right index finger; in the other session, they tapped with the right big toe. In order to control for possible carryover effects, half the subjects tapped with the finger first and with the toe second; the other half tapped in reverse order. Each session was subdivided into two blocks of 10 trials. In the first block, no additional feedback was presented. In the second block, an auditory signal was presented over the headphones each time the subject touched the response key. The subjects were instructed to concentrate on this additional auditory signal and to synchronize it with the auditory pacing signal. The procedure within trials was the same as in Experiment 1. At the beginning of each session, the subjects ran through seven warm-up trials (under Condition tk).

\section{Results}

The data elimination procedure was identical to that in Experiment 1 . Overall, $1 \%$ of the trials had to be rejected. The means of the 20 asynchronies per trial were entered into an ANOVA that distinguished three withinsubject factors: effector (hand vs. foot), feedback (tk vs. $\mathrm{tk}+\mathrm{a})$, and repetition within blocks ( 10 trials per block). Three sources of variance were significant: the main effects of effector $[F(1,13)=10.66, p<.01]$ and feedback $[F(1,13)=12.43, p<.01]$, and their interaction effector $\times$ feedback $[F(1,13)=11.62, p<.01]$. Because there was no main effect of repetitions and there were no interactions involving this factor, only the averages of the 10 repetitions were considered in further analyses.

As in Experiment 1, a negative asynchrony was observed when the hand performed the movement $(\bar{X}=$ $-25 \mathrm{msec}, s=17 \mathrm{msec}$ ) and a larger asynchrony when the foot performed the movement $(\bar{X}=-53 \mathrm{msec}, s=$ $33 \mathrm{msec}$ ). When additional auditory feedback was provided, the asynchrony was smaller than in the tk feedback condition (tk $+\mathrm{a}, \vec{X}=-31 \mathrm{msec}, s=19 \mathrm{msec}$; tk, $\bar{X}=-48 \mathrm{msec}, s=25 \mathrm{msec}$ ). As the significant interaction indicated, the size of this effect differed for the hand and the foot condition. In the hand condition, there

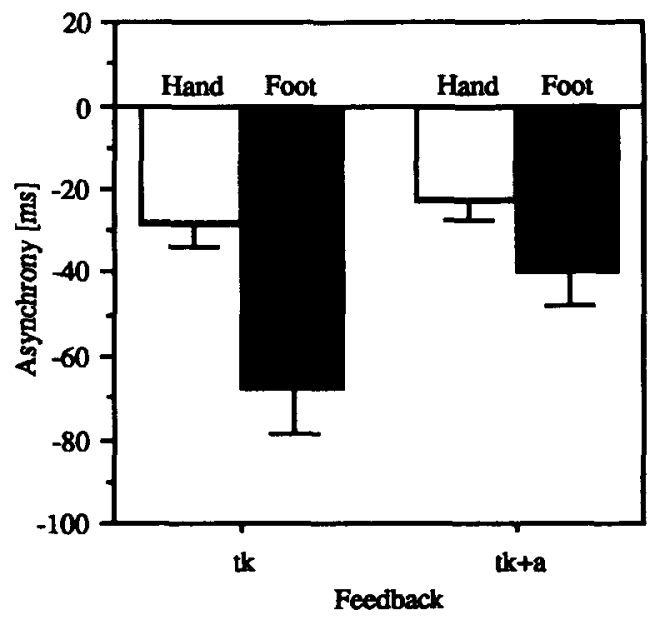

Figure 5. Mean negative asynchrony in two feedback conditions (tk: only tactile/kinesthetic feedback, tk+a: additional auditory feedback) under two effector conditions (hand vs. foot).

was only a slight decrease of the asynchrony when additional feedback was administered. Although very small $(6 \mathrm{msec})$, this difference was significant $[t(13)=2.9$, $p<.05]$. In the foot condition, the effect was much more pronounced ( $28 \mathrm{msec}$; see Figure 5).

Again, the standard deviation of the asynchrony was larger when the foot performed the movement than it was in the hand condition (foot, $38 \mathrm{msec}$; hand, $32 \mathrm{msec}$ ). The difference between the two feedback conditions was not significant, nor was the interaction of both factors.

As in Experiment 1, the key-touching time was larger in the foot than in the hand condition (foot, $202 \mathrm{msec}$; hand, $153 \mathrm{msec}$ ). When additional auditory feedback was provided, the key-touching time was smaller than in the tk feedback condition (tk, $213 \mathrm{msec}$; tk+a, $158 \mathrm{msec}$ ). No interaction was observed.

\section{Discussion}

Again, the results support the Paillard-Fraisse hypothesis. The fact that the asynchrony is reduced but does not drop to zero under conditions with additional feedback supports the third of the theoretical options discussed above: The two sources of feedback enter into a joint-event code whose effective onset point is computed as a weighted average from the onset points of its two components. The mixture-of-trials hypothesis mentioned above is clearly ruled out after a closer examination of the variances. It would predict an increase in variance when additional auditory feedback is provided - either between subjects or between trials or within trials. At none of these levels was an increase of variance observed; variances were either constant or even reduced under conditions with auditory feedback. We can therefore reject the mixture-of-trials hypothesis and retain the notion of a joint-event code.

The significant interaction of effector $\times$ feedback supports the idea of a weighted average. The sensory codes 
representing both kinds of feedback information (tactile/kinesthetic and auditory) determine the generation of the common event code. If auditory feedback acts as an additive constant, no interaction should be observed, because there would be a reduced amount of negative asynchrony under both effector conditions. However, if each of the feedback codes is weighted with a factor, it should result in the observed interaction between the factors effector and feedback, because weighting a greater amount of asynchrony (in the foot condition) with the same factor as that for a smaller amount of asynchrony leads to a greater reduction of asynchrony (than in the hand condition).

As for the values of the asynchrony in the various conditions, the difference between hand and foot tapping is the same as that reported in the literature. Under conditions in which additional feedback was eliminated (tk condition), a difference between hand and foot tapping of $40 \mathrm{msec}$ to $45 \mathrm{msec}$ was found. These values correspond to results reported in EEG experiments (e.g., Shibasaki et al., 1981; see Experiment 1). In conditions that were comparable to the tk+a condition in the reported experiment, the difference between hand and foot tapping was clearly reduced to values of $10 \mathrm{msec}$ to $20 \mathrm{msec}$ (Bard et al., 1992; Fraisse, 1980; Paillard, 1949).

\section{EXPERIMENT 3}

In Experiment 2, a reduction of the asynchrony under the $\mathrm{tk}+\mathrm{a}$ conditions was observed, but the asynchrony still remained. Under these conditions, the subjects had to produce taps in such a way that two different auditory stimuli (the pacing signal, a $400-\mathrm{Hz}$ tone; and the feedback signal, a $2000-\mathrm{Hz}$ tone) appeared simultaneously. This suggests the possibility that the observed effects might have been due to purely sensory factors within the auditory modality.

As is known from the masking literature, the audibility threshold for one sound (test stimulus) is raised by the presence of another sound (masking stimulus), whereby the masking stimulus can be presented before the test stimulus (forward masking) or afterward (backward masking). Moreover, the interaction between two auditory stimuli is greatly influenced by a variety of factors, such as loudness, pitch, and duration of test and masking tone. For example, when a subject is asked to identify the pitch of a tone, performance usually improves as a negatively accelerated function of the intertone interval (Hawkins, Thomas, Presson, Cozic, \& Brookmire, 1974; Massaro, 1970; Massaro \& Idson, 1978). Moreover, forward and backward masking have different effects on test stimuli (Elliott, 1971; Lynn \& Small, 1977; Pastore, Harris, \& Goldstein, 1980).

Since both types of masking might have occurred in Experiment 2, the asynchronies observed in the auditory feedback conditions might have been influenced by purely sensory factors within the auditory modality. There is a possibility that, owing to masking effects, the perceived synchrony of the two auditory signals can be obtained only when their onsets are noncoincident.

In the auditory feedback condition the asynchrony was clearly dependent on the effector used, so we may argue that the masking interpretation is weakened since this difference cannot be due to purely sensory factors within the auditory modality. Nevertheless, it does not completely rule out masking effects that might still influence the size of the asynchrony in both effector conditions.

A further consideration pertains to individual differences in synchronization. In the experiments reported so far, we observed quite substantial individual differences in the size of the asynchrony. For instance, in the auditory feedback condition of Experiment 2, the mean asynchronies for hand tapping ranged between $-3 \mathrm{msec}$ and $-56 \mathrm{msec}$. Since the task required the synchronization of the appearance of the two stimuli, one possible explanation of these large differences might be that there were individual differences in simultaneity judgment.

The following control experiment was run, first, in order to examine how precisely subjects would be able to tell whether the two auditory stimuli used in Experiment 2 occurred simultaneously or not and to what extent individual differences would emerge. In this experiment, the two auditory stimuli were presented at various SOAs, and the subjects were asked to judge whether they had occurred simultaneously or not. Second, if purely sensory interactions between the two auditory stimuli are reflected in the asynchronies, one would not expect the best impression of synchrony to be obtained with synchronous stimulus onsets but rather at a certain amount of SOA.

\section{Method}

Subjects. Twelve new subjects ( 4 female and 8 male, mean age 25 years) participated in the experiment. All had normal auditory acuity and were naive with regard to the experiment's purpose.

Apparatus and Stimuli. The subject sat at a table in a soundabsorbing room. The same auditory signals as in Experiment 2 $(400 \mathrm{~Hz}$ and $2000 \mathrm{~Hz}, 82 \mathrm{~dB}[\mathrm{~A}], 10 \mathrm{msec})$ were presented binaurally through headphones (AKG K240,600 $\Omega$ ). They were produced by a personal computer (Hewlett-Packard Vectra QS/20) via a D/A converter and an amplifier (Sony TA-F170). The computer controlled the experimental procedure and registered the responses.

Procedure. The SOA between the two signals, the $400-\mathrm{Hz}$ tone and the $2000-\mathrm{Hz}$ tone, was varied in 20 steps ranging from -127 to $+127 \mathrm{msec}$ (negative values indicate that the $2000-\mathrm{Hz}$ tone comes first; the $20 \mathrm{SOA}$ values were $\pm 127, \pm 95, \pm 71, \pm 53, \pm 35, \pm 23, \pm 15$, $\pm 10, \pm 5, \pm 2$ ). No stimuli were presented with 0 -msec separation, because a pilot study had shown that precise physical synchrony is always detected and can therefore be used by the subjects as the sole criterion for perceived synchrony. The graduation of the SOA values is finer around $0 \mathrm{msec}$ than around $100 \mathrm{msec}$; for example, the distance between the highest values is $33 \mathrm{msec}$, whereas it is $3 \mathrm{msec}$ around $0 \mathrm{msec}$.

The experimental procedure consisted of 60 blocks; each of the 20 SOA values was used three times as a starting SOA. Each block consisted of several trials, and, within one trial, the sequence of 10 pairs of signals was presented. The exact number of trials within one block depended on the point in time when the subject judged 
simultaneity. The sequence of the SOA values within one block was either ascending (in blocks with a negative starting value) or descending (in blocks with a positive value).

The subjects had to judge pairs of tones that were presented in trials. Each trial consisted of a sequence of 10 pairs of signals presented with a constant SOA. As in Experiment 2, the interval between the $400-\mathrm{Hz}$ signals (the former pacing signal) was $800 \mathrm{msec}$. After listening to the trial, the subjects were asked to judge whether the signals had been simultaneous or not (yes or no, respectively). When the subject pressed the yes key, the next block with a different SOA was started, which was randomly chosen. When the subject pressed the no key, another trial with the next smaller SOA was started, was judged by the subjects, and so on. The subject was instructed to judge yes as soon as both stimuli seemed to be simultaneous, because the absolute value of the SOA would increase again if the subject missed the point of objective simultaneity. A new block with a new SOA value (selected randomly) was started only when the subject pressed the yes key.

At the very beginning of the session, the subjects underwent a demonstration phase in which they listened to 20 trials on which each SOA value was presented once. Subsequently, the subjects were made familiar with the apparatus in two practice trials.

\section{Results}

In Figure 6, the psychophysical function for all subjects is displayed. As the function is stable below a SOA of $-35 \mathrm{msec}$ (the cumulative frequency of simultaneity judgment is $0 \%$ ) and above $+35 \mathrm{msec}$ (the cumulative frequency of simultaneity judgment is $100 \%$ ) Figure 6 is limited to these values, although the range of the SOAs presented was larger $(-127 \mathrm{msec}$ to $+127 \mathrm{msec})$. The point of subjective simultaneity (the $50 \%$ point) was almost identical with the point of objective synchrony. This basic pattern was observed for each individual subject. The point of subjective simultaneity ranged from $-3 \mathrm{msec}$ to $+5 \mathrm{msec}$ between subjects.

\section{Discussion}

This control experiment shows that subjects are able to perceive very precisely whether or not the stimuli used in Experiment 2 appear simultaneously. Furthermore, since the psychophysical function is highly symmetrical, there is no tendency to prefer one over the other

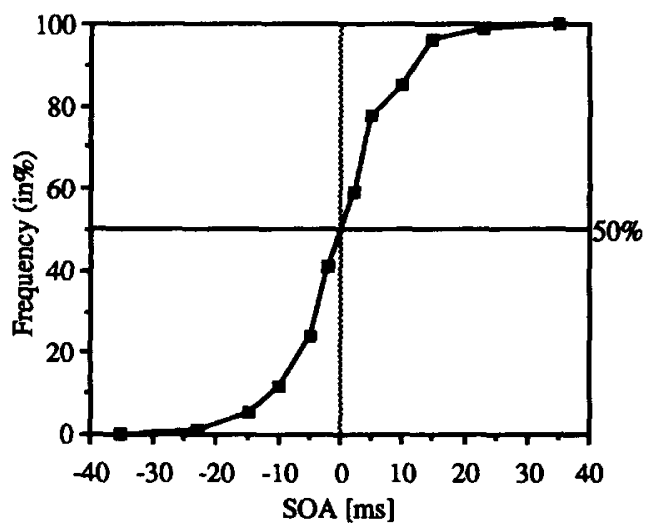

Figure 6. Cumulative frequency (in percent) of simultaneity judgments at a given stimulus onset asynchmony (SOA). sequence of tones. It must be concluded that simple sensory interactions between the two auditory stimuli cannot be responsible for the observed asynchrony.

This also holds for individual differences which are very small, ranging from $-3 \mathrm{msec}$ to $+5 \mathrm{msec}$ for the $50 \%$ point, and cannot account for the huge interindividual differences usually observed in synchronization tasks.

It must therefore be concluded that the negative asynchrony is due to factors that reflect functional features of the synchronization task. One such factor is the involvement of voluntary movement--that is, the finger movement and its accompanying tactile/kinesthetic feedback. The Paillard-Fraisse hypothesis assumes that different nerve conduction times are responsible for the asynchrony. Under this view, the reported results confirm our expectations. Because the subject's task is to produce synchrony between two auditory stimuli, nerve conduction and central processing times should be almost identical. In this situation, synchrony of sensory codes at the central level should also lead to synchrony of the external events that are represented by these sensory codes.

\section{GENERAL DISCUSSION}

The results lend clear support to the Paillard-Fraisse hypothesis. First, negative asynchronies are observed throughout, replicating previous findings in the literature. Second, asynchronies are clearly larger for foot than for hand tapping, as is anticipated by the hypothesis that nerve conduction time is a critical factor in the underlying mechanism. Third, as must be expected on the same account, negative asynchronies become shorter when auditory feedback is provided in addition to the tactile/kinesthetic feedback available from the tap itself. These results support the conclusion that synchrony is established at the level of sensory coding of clicks and taps. Synchrony is achieved by timing the taps in such a way that, at the level of common sensory coding, tap codes coincide in time with click codes.

One might expect that an account like this, which strongly relies on the system's hardware features, does not leave much room for individual differences and is therefore weakened to the extent that such differences occur. In fact, substantial individual differences were observed in all experiments. They refer to both the absolute size of asynchronies (ranging, e.g., from $-11 \mathrm{msec}$ to $-90 \mathrm{msec}$ in the hand-tapping condition of Experiment 1) and their susceptibility to experimental manipulations (e.g., hand-foot differences ranging from $26 \mathrm{msec}$ to $70 \mathrm{msec}$ in Experiment 1). Yet, as physiological evidence indicates, this is in reasonable agreement with the magnitude of individual differences in peripheral conduction times. For example, EEG experiments also show large interindividual differences. Shibasaki et al. (1981) measured evoked potentials in subjects while they were tapping with the foot. The "post-motion frontal negativity," which can be interpreted as an indicator for kinesthetic feedback, occurred 50-160 msec after the EMG peak. 
This suggests that the Paillard-Fraisse hypothesis is valid; but before we definitely draw this conclusion, we will first examine the extent to which the competing hypotheses mentioned in the introduction could account for the results as well. In order to examine the P-center hypothesis, we will consider the relationships between asynchronies and key-touching times under the different experimental conditions. To test the evaluation hypothesis, we will likewise consider how the means of asynchronies are related to their standard deviations under different conditions. ${ }^{2}$

As was mentioned in the introduction, the evaluation hypothesis predicts that the size of asynchrony must depend on the variance of tap generation. It is reasonable to assume that this variance might be larger for foot than for hand tapping. Yet, in order to account for the substantial difference in the mean asynchronies $(45 \mathrm{msec}$, Experiment 1; $28 \mathrm{msec}$, Experiment 2), the standard deviations would have to show a difference of the same order of magnitude. This was clearly not the case in both experiments. Although the standard deviations tended to be slightly larger for the foot than for the hand conditions (a difference of $2 \mathrm{msec}$ in Experiment 1; of $6 \mathrm{msec}$ in Experiment 2), the size of the effect was by far not sufficient to account for the hand-foot differences in the mean asynchronies. The same argument applies to the feedback manipulation, in which a substantial effect in the asynchronies $(17 \mathrm{msec})$ was not accompanied by a corresponding difference in the standard deviations $(-2 \mathrm{msec})$. As a result, we have to conclude that the evaluation hypothesis cannot account for the results and is thus ruled out.

According to the $\mathrm{P}$-center hypothesis, the asynchronies reflect the fact that the tap's functional onset lags behind its physical onset. This lag should, among other things, depend on the tap's total duration (key-touching time): The longer the finger touches the key, the more its functional onset should lag behind its physical onset, and the larger the asynchrony should be. In Experiment 1, some aspects of the results are compatible with this account and some are not. On the one hand, if one compares the asynchronies and key-touching times from the hand and foot conditions, a similar ratio is observed in both cases (.37 for the hand conditions and .44 for the foot conditions). This might support the notion that asynchronies depend on tap durations and that this relationship is mediated through the location of the functional onset point. However, in the same experiment, we also find evidence to the contrary. Key-touching times for the hand conditions were considerably smaller under horizontal coupling than under vertical and diagonal coupling (130 msec vs. $171 / 178 \mathrm{msec}$ ), but there was not the slightest indication of a related difference in the asynchronies (cf. Figure 3 ).

The control condition of Experiment 2 (tactile/kinesthetic feedback only) can to some extent be considered a replication of Experiment 1. In this experiment, however, there was no constancy in the ratio between asynchronies and key-touching times for the two effector conditions ( .15 for the hand and .28 for the foot). Moreover, a clear interaction between the feedback conditions and the effector conditions was observed in the asynchronies, but not in the key-touching times. This implies that the P-center hypothesis fails to account for this pattern of results (at least unless additional assumptions are introduced).

On the other hand, since this interaction is clearly predicted by the Paillard-Fraisse hypothesis, we have to conclude that this hypothesis is, for the time being, the sole survivor in this competition. We will therefore briefly examine some of its major theoretical implications that refer to periodic synchronization proper as well as to the relationship between perception and action in general.

One of the implications of the Paillard-Fraisse hypothesis is that a system operating on the principle of temporal coincidence of brain codes cannot-by that very principle-achieve temporal coincidence of the coded events themselves. This raises the question of why the system should not be efficient enough to establish veridical event coincidence (in the world) and why it should stick to the less efficient principle of code coincidence (in the brain) instead. A tentative answer is that achieving event coincidence is computationally much more demanding than achieving brain code coincidence. As can be derived from Figure 1, veridical event coincidence would require the system to maintain certain delays between the brain codes involved in generating and in representing the respective events. No such delays are required under the principle of brain code coincidence. It is reasonable to assume that superimposing two codes in time is computationally less demanding than keeping them separate by fixed amounts of time. Though establishing brain code coincidence is simpler than establishing world event coincidence, veridicality is the price to be paid for that simplicity.

On the other hand, people are, in many daily life situations, capable of attaining true event coincidence (e.g., in catching a ball or in shooting at a flying bird). The critical prerequisite for this seems to be that the task provides unequivocal feedback about the motor act's effect (e.g., having caught the ball or having hit the bird). Such feedback should help one overcome the computational problems involved in achieving event coincidence. However, in the periodic synchronization task applied in our experiments, such unequivocal feedback is not available. In this task, there is nothing comparable to the catching of a ball or the hitting of a bird. Rather, the perceived simultaneity of clicks and taps is the sole source of feedback available. However, this source of information seems to be too unreliable to account for the high-precision computations required to achieve event coincidence. This can be derived from the fact that subjects tend to perceive perfect synchrony between taps and clicks, even if they are generating substantial asynchronies. ${ }^{3}$

We may therefore assume that under conditions that lack unequivocal feedback, the system sticks to operating on the simple principle of code coincidence and does 
not switch to the more complex computations required for event coincidence, unless such feedback is available. According to this view, brain-code coincidence is considered a fallback option that is available to the system whenever the feedback requirements for achieving worldevent coincidence are not fulfilled. Further studies including unequivocal feedback are needed in order to test this view.

A further implication of the Paillard-Fraisse hypothesis is also related to the computational simplicity of code coincidence: This principle can easily account for one of the particularly remarkable features of synchronization performance that has attracted the interest of many researchers-namely, that synchronizing periodic stimuli is a child's game. The task stands out from the vast majority of sensorimotor tasks by the fact that virtually no learning is necessary in order to establish satisfactory performance. The periodic pattern is easy to capture, and it is just as easy to anticipate its continuation in the immediate future. Subjects are more or less perfect from the outset of each trial. It appears that the periodic pattern of stimulation serves as a sufficient basis for the specification of the required motor acts; that is, it is not only of indicational but also of specificational use in the guidance of the pattern of action (cf. Kelso \& Kay, 1987; Pattee, 1974).

Finally, the perhaps most general implication of the Paillard-Fraisse hypothesis seems to be that it may shed new light on the nature of the perception-action interface. Traditional views of the relationship between perception and action tend to stress the incommensurate nature of sensory codes and motor commands, invoking the notion of two separate coding domains as well as translation-like processes between them (see, e.g., Massaro, 1990; Sanders, 1980; Welford, 1968, 1980). Instead, the notion of code coincidence inherent in the PaillardFraisse hypothesis suggests a view that stresses the commensurability of actions and events in terms of their entry into a common representational domain and, therefore, the role of action effects (both perceived and expected) in action control (see, e.g., Allport et al., 1987; Greenwald, 1970; Meltzoff et al., 1991; Prinz, 1990, 1992).

\section{REFERENCES}

Allport, A., Mackay, D. G., Prinz, W., \& Scheerer, E. (Eds.) (1987). Language perception and production. London: Academic Press.

AsCHERSLEBEN, G. (1994). Afferente Informationen und die Synchronisation von Ereignissen [Afferent information and the synchronization of events]. Frankfurt: Lang.

Auxiette, C. (1992). Coordination et synchronisation de deux sequences sonores perçues et produites [The coordination and synchronization of two perceived and produced sound sequences]. Unpublished doctoral dissertation, University of Paris.

Bard, C., Paillard, J., Lajoie, Y., Fleury, M., Teasdale, N., ForGET, R., \& LAMARRE, Y. (1992). Role of the afferent information in the timing of motor commands: A comparative study with a deafferent patient. Neuropsychologia, 30, 201-206.

Bard, C., Paillard, J., Teasdale, N., Fleury, M., \& Lajoie, Y. (1991). Self-induced versus reactive triggering of synchronous hand and heel movement in young and old subjects. In J. Requin \& G. E. Stelmach (Eds.), Tutorials in motor neuroscience (pp. 189-196). Amsterdam: Kluwer.

Collyer, C. E., Broadbent, H. A., \& Church, R. M. (1992). Categorical time production: Evidence for discrete timing in motor control. Perception \& Psychophysics, 51, 134-144.

Collyer, C. E., Broadbent, H. A., \& Church, R. M. (1994). Preferred rates of repetitive tapping and categorical time production. Perception \& Psychophysics, 55, 443-453.

DenNetT, D. C., \& Kinsbourne, M. (1992). Time and the observer: The where and the when of consciousness and the brain. Behavioral \& Brain Sciences, 15, 183-247.

DunLaP, K. (1910). Reactions on thythmic stimuli, with attempt to synchronize. Psychological Review, 17, 399-416.

ELLIOTT, L. L. (1971). Backward and forward masking. Audiology, 10, 65-76.

Fraisse, P. (1966). L'anticipation de stimulus rythmiques, vitesse d'établissement et précision de la synchronisation [Anticipation of rhythmical stimuli, set-up speed and accuracy of synchronization]. L'Année Psychologique, 66, 15-36.

FRAISSE, P. (1980). Les synchronisations sensori-motrices aux rythmes [The sensorimotor synchronization of rhythms]. In J. Requin (Ed.), Anticipation et comportement (pp. 233-257). Paris: Centre National.

Fraisse, P., Oléron, G., \& Paillard, J. (1958). Sur les repères sensoriels qui permettent de contrôler les mouvements d'accompagnement de stimuli périodiques [On the sensory reference points that allow for controlling movements accompanying periodic stimuli]. L'Année Psychologique, 58, 322-338.

FraneK, M., Radil, T., Indra, M., \& LánSKy, P. (1987). Following complex rhythmical acoustical patterns by tapping. International Journal of Psychophysiology, 5, 187-192.

GeIsSER, S., \& Greenhouse, S. W. (1958). An extension of Box's results on the use of F-distribution in multivariate analysis. Annals of Mathematical Statistics, 29, 885-891.

GérARD, C., \& AUXIETTE, C. (1992). The processing of musical prosody by musical and nonmusical children. Music Perception, 10, 93-126.

GREenWALD, A. G. (1970). Sensory feedback mechanisms in performance control: With special reference to the ideo-motor mechanism. Psychological Review, 77, 73-99.

HARY, D., \& MooRE, G. P. (1985). Temporal tracking and synchronization strategies. Human Neurobiology, 4, 73-77.

HARY, D., \& MOORE, G. P. (1987). Synchronizing human movement with an external clock source. Biological Cybernetics, 56, 305-311.

Hawkins, H. L., Thomas, G., Presson, J., Cozic, A., \& Brookmire, D (1974). Precategorical selective attention and tonal specificity in auditory recognition. Journal of Experimental Psychology, 103, $530-538$

Hirsh, I. J., \& ShERrick, C. E. (1961). Perceived order in different sense modalities. Journal of Experimental Psychology, 62, 423-432.

Ilmberger, J., Müller, U., PöPPEl, E., MATes, J., \& RAdIL, T. (1990). Timing of rhythmic tapping and lateralization. Activas Nervosa Superior, 32, 142.

IVRY, R. B., KeELE, S. W., \& Diener, H. C. (1988). Dissociation of the lateral and medial cerebellum in movement timing and movement execution. Experimental Brain Research, 73, 167-180.

Kelso, J. A. S., \& KAY, B. A. (1987). Information and control: A macroscopic analysis of perception-action coupling. In $\mathrm{H}$. Heuer \& A. F. Sanders (Eds.), Perspectives on perception and action (pp. 3-32). Hillsdale, NJ: Erlbaum.

КосH, R. (1992). Sensumotorische Synchronisation: Eine Kostenanalyse [Sensorimotor synchronization: A cost analysis] (Paper 11/92). Munich: Max-Planck-Institute for Psychological Research.

Kolers, P. A., \& Brewster, J. M. (1985). Rhythms and responses. Journal of Experimental Psychology: Human Perception \& Performance, 11, 150-167.

LYNN, G. K., \& SMALL, A. M. (1977). Interactions of backward and forward masking. Journal of the Acoustical Society of America, 61 , 185-189.

Marcus, S. M. (1976). Perceptual centres. Unpublished doctoral dissertation, Cambridge University. 
Massaro, D. W. (1970). Preperceptual auditory images. Journal of Experimental Psychology, 85, 411-417.

Massaro, D. W. (1990). An information-processing analysis of perception and action. In O. Neumann \& W. Prinz (Eds.), Relationships between perception and action: Current approaches (pp. 133-166). Berlin: Springer-Verlag.

Massaro, D. W., \& IDSON, W. L. (1978). Target-mask similarity in backward recognition masking of perceived tone duration. Perception \& Psychophysics, 24, 225-236.

MATES, J. (1991). Extending the model of self-paced periodic responding: Comment on Vos and Ellermann (1989). Journal of Experimental Psychology: Human Perception \& Performance, 17, 877-879.

MATES, J. (1994a). A model of synchronization of motor acts to a stimulus sequence: I. Timing and error corrections. Biological $\mathrm{Cy}$ bernetics, 70, 463-473.

MAtes, J. (1994b). A model of synchronization of motor acts to a stimulus sequence: II. Stability analysis, error estimation and simulations. Biological Cybernetics, 70, 475-484.

Mates, J., Radil, T., \& Pöppel, E. (1992). Cooperative tapping: Time control under different feedback conditions. Perception \& Psychophysics, 52, 691-704.

Meltzoff, A. N., Kuhl, P. K., \& Moore, M. K. (1991). Perception, representation, and the control of action in newborns and young infants: Towards a new synthesis. In M. J. Weiss \& P. R. Zelazo (Eds.), Newborn attention: Biological constraints and the influence of experience (pp. 377-411). Norwood, NJ: Ablex.

Morton, J., Marcus, S. M., \& Frankish, C. (1976). Perceptual centers (P-centers). Psychological Review, 83, 405-408.

NAJENSON, T., RON, S., \& BEHROOZI, K. (1989). Temporal characteristics of tapping responses in healthy subjects and in patients who sustained cerebrovascular accident. Brain, Behavior \& Evolution, 33, 175-178.

OlÉRON, G. (1961). Attitude et utilisation des repères dans la synchronisation de stimuli périodiques [The role and the use of reference points in the synchronization of periodic stimuli]. L'Année Psychologique, 61, 59-78.

Paillard, J. (1949). Quelques données psychophysiologiques relatives au déclenchement de la commande motrice [Some psychophysiological data relating to the triggering of motor commands]. L'Année Psychologique, 48, 28-47.

Pastore, R. E., Harris, L. B., \& Goldstein, L. (1980). Auditory forward and backward masking interaction. Perception \& Psychophysics, 28, 547-549.

Pattee, H. H. (1974). Discrete and continuous processes in computers and brains. In M. Conrad, W. Guttinger, \& M. D. Cin (Eds.), Physics and mathematics of the nervous system (pp. 128-148). New York: Springer-Verlag.

PETERS, M. (1989). The relationship between variability of intertap intervals and interval duration. Psychological Research, 51, 38-42.

PöPPel, E., Müller, U., \& MATES, J. (1990, October). Temporal constraints in synchronization of motor responses to a regular sequence of stimuli. Paper presented at the annual meeting of the Society for Neuroscience, St. Louis.

Povel, D. J. (1981). Internal representation of simple temporal patterns. Journal of Experimental Psychology: Human Perception \& Performance, 7, 3-18.

PRINZ, W. (1990). A common coding approach to perception and action. In O. Neumann \& W. Prinz (Eds.), Relationships between perception and action: Current approaches (pp. 167-201). Berlin: Springer-Verlag.

PRINZ, W. (1992). Why don't we perceive our brain states? European Journal of Cognitive Psychology, 4, 1-20.

RutschmanN, J., \& Link, R. (1964). Perception of temporal order of stimuli differing in sense mode and simple reaction time. Perceptual \& Motor Skills, 18, 345-352.

SANDERS, A. F. (1980). Stage analysis of reaction processes. In G. E. Stelmach \& J. Requin (Eds.), Tutorials in motor behavior (pp. 331354). Amsterdam: North-Holland.

SCHULZE, H.-H. (1992). The error correction model for the tracking of a random metronome: Statistical properties and an empirical test.
In F. Macar, V. Pouthas, \& W. J. Friedman (Eds.), Time, action and cognition (pp. 275-286). Dordrecht: Kluwer.

SCHÜtTE, H. (1978a). Ein Funktionsschema für die Wahrnehmung eines gleichmäßigen Rhythmus in Schallimpulsen [A functional scheme for perceiving a regular rhythm in auditory impulses]. Biological Cybernetics, 29, 49-55.

SCHÜTTE, H. (1978b). Subjektiv gleichmäßiger Rhythmus: Ein Beitrag zur zeitlichen Wahrnehmung von Schallereignissen [Subjective regular rhythm: A contribution to the temporal perception of auditory events]. Acustica, 41, 197-206.

Shibasaki, H., Barrett, G., Halliday, E., \& Halliday, A. M. (1981). Cortical potentials associated with voluntary foot movement in man. Electroencephalography \& Clinical Neurophysiology, 52, 507-516.

Sternberg, S., \& KNoll, R. L. (1973). The perception of temporal order: Fundamental issues and a general model. In S. Kornblum (Ed.), Attention and performance IV (pp. 629-685). New York: Academic Press.

Stevens, L. T. (1886). On the time sense. Mind, 11, 393-404.

STONE, S. A. (1926). Prior entry in the auditory-tactual complication. American Journal of Psychology, 37, 284-287.

Summers, J. J., Bell, R., \& Burns, B. D. (1989). Perceptual and motor factors in the imitation of simple temporal patterns. Psychological Research, 50, 23-27.

TerhardT, E., \& SchütTe, H. (1976). Akustische RhythmusWahrnehmung: Subjektive Gleichmäßigkeit [Acoustical rhythm perception: Subjective regularity]. Acustica, 35, 122-126.

Truman, G., \& Hammond, G. R. (1990). Temporal regularity of tapping by the left and right hands in timed and untimed finger tapping. Journal of Motor Behavior, 22, 521-535.

VorberG, D., \& Hambuch, R. (1978). On the temporal control of rhythmic performance. In J. Requin (Ed.), Attention and performance VII (pp. 535-555). Hillsdale, NJ: Erlbaum.

VORBERG, D., \& HAMBUCH, R. (1984). Timing of two-handed rhythmic performance. In J. Gibbon \& L. Allan (Eds.), Timing and time perception (Annals of the New York Academy of Sciences, Vol. 423, pp. 390-406). New York: New York Academy of Sciences.

VoRBERG, D., \& WING, A. (1993). Modelle für die Variabilität und Abhängigkeit bei der zeitlichen Steuerung [Modeling variability and dependence in timing]. In H. Heuer \& S. Keele (Eds.), Enzyklopädie der Psychologie, Serie Kognition: Band III. Psychomotorik (pp. 223320). Göttingen: Hogrefe.

Vos, P. G., \& EllermanN, H. H. (1989). Precision and accuracy in the reproduction of simple tone sequences. Journal of Experimental Psychology: Human Perception \& Performance, 15, 179-187.

Vos, P. G., \& HELSPER, E. L. (1992). Tracking simple rhythms: On-beat versus off-beat performance. In F. Macar, V. Pouthas, \& W. J. Friedman (Eds.), Time, action and cognition (pp. 287-299). Dordrecht Kluwer.

Vos, P. G., Helsper, E. L., \& van Krysbergen, N. (1992). Tracking simple rhythms under pseudo-synchronization conditions. In Proceedings of the Fourth Rhythm Workshop: Rhythm Perception and Production (pp. 37-42). Bourges: CNRS.

WELFORD, A. T. (1968). Fundamentals of skill. London: Methuen. Welford, A. T. (1980). Reaction times. London: Academic Press. WING, A. M. (1977). Perturbations of auditory feedback delay and the timing of movement. Journal of Experimental Psychology: Human Perception \& Performance, 3, 175-186.

WING, A. M. (1982). Timing and co-ordination of repetitive bimanual movements. Quarterly Journal of Experimental Psychology, 37A, 339-348.

Wing, A. M., \& Kristofferson, A. B. (1973a). Response delays and the timing of discrete motor responses. Perception \& Psychophysics, 14, 5-12.

Wing, A. M., \& Kristofferson, A. B. (1973b). The timing of interresponse intervals. Perception \& Psychophysics, 13, 455-460.

WoODROw, H. (1932). The effect of rate of sequence upon the accuracy of synchronization. Journal of Experimental Psychology, 15, 357-379.

Yamanishi, J., KaWato, M., \& SUZUKI, R. (1979). Studies on human finger tapping neural networks by phase transition curves. Biological Cybernetics, 33, 199-208. 
Yamanishi, J., Kawato, M., \& SuzUki, R. (1980). Two coupled oscillators as a model for the coordinated finger tapping by both hands. Biological Cybernetics, 37, 219-225.

\section{NOTES}

1. In order to avoid the risk of violating statistical assumptions in repeated-measures designs due to the inhomogeneity of the variancecovariance matrix, $p$ values were corrected according to Geisser and Greenhouse (1958).

2. No such criterion is available for the undershooting hypothesis. In order to obtain such a criterion one would have to specify (1) how the experimental variables manipulated would affect the variance of tap asynchronies in the absence of tradeoff and (2) the tradeoff function that specifies how the timekeeper's variance depends on the interval to be controlled.

3. This is not at variance with the high precision of simultaneity judgments observed in Experiment 3, in which subjects produced intramodal judgments within the auditory modality. Precision is much lower when judgments refer to the temporal relationships of events across modalities (Hirsh \& Sherrick, 1961; Rutschmann \& Link, 1964; Sternberg \& Knoll, 1973; Stone, 1926).

(Manuscript received March 23, 1994; revision accepted for publication September 27, 1994.) 